Instituto Internacional de Investigación y Desarrollo Tecnológico Educativo INDTEC, C.A.

DOI: https://doi.org/10.29394/Scientific.issn.2542-2987.2018.3.7.6.121-139

OAI-PMH: http://www.indteca.com/ojs/index.php/Revista Scientific/oai

\title{
Programa de Reforestación como Alternativa Ambiental en la Recuperación de Espacios de Recreación y Esparcimiento
}

Autora: Emerita Molina Contreras

Universidad Pedagógica Experimental Libertador, UPEL

emeritacontreras71@gmail.com

Barinas, Venezuela

\section{Resumen}

La presente investigación tiene como propósito desarrollar un programa de reforestación como alternativa ambiental en la recuperación de espacios de recreación y esparcimiento en la Comunidad Morrocoy Abajo sector I, Parroquia San Silvestre, Municipio Barinas, Estado Barinas. En lo que refiere. Al aspecto metodológico, el estudio se enfoca en el paradigma cualitativo, teniendo como apoyo el método etnográfico, se considera como técnica a utilizar la entrevista, en cuanto a los informantes claves se consideraron tres (03), los cuales, fueron elegidos a criterio de la investigadora, según los actores que se consideran vinculantes: vecinos conocedores de la materia ambiental pertenecientes a la comunidad en estudio. Como conclusión, se puede hacer mención al hecho de que la investigación se encuentra actualmente en proceso de desarrollo, pudiendo establecer que, las comunidades organizadas, manejan estrategias específicas para la integración de estas, donde la orientación oportuna como un proceso social conlleva a la participación activa y efectiva de los involucrados; de allí, este planteamiento, señala que el proceso de participación comunitaria debe ser pertinente a las necesidades del individuo-sociedad donde se lleve a cabo. forestal.

Palabras clave: educación ambiental; medio ambiente; gestión

Fecha de Recepción: 17-06-2017 Fecha de Aceptación: 07-10-2017 


\title{
Reforestation Program as an Environmental Alternative in the Recovery of Recreation and Leisure Spaces
}

\begin{abstract}
The purpose of this research is to develop a reforestation program as an environmental alternative in the recovery of recreational and leisure spaces in the Morrocoy Abajo Community, Sector I, San Silvestre Parish, Barinas Municipality, Barinas State. In what refers. To the methodological aspect, the study focuses on the qualitative paradigm, having as support the ethnographic method, it is considered as a technique to use the interview, as for the key informants, three (03) were considered, which were chosen according to the criterion of the researcher, according to the actors that are considered binding: neighboring knowledgeable of the environmental matter belonging to the community under study. As a conclusion, mention can be made of the fact that the research is currently in the process of being developed, being able to establish that organized communities handle specific strategies for the integration of these, where timely guidance as a social process leads to participation active and effective of those involved; from there, this approach indicates that the process of community participation must be relevant to the needs of the individual-society where it is carried out.
\end{abstract}

Keywords: environmental education; environment; forest management. 


\section{Introducción}

El deterioro ambiental es observable en el contexto mundial, de allí que existan especies en peligro de extinción, contaminación, tala y quema de bosques, acelerado crecimiento poblacional, biodiversidades amenazadas, degradación de hábitats, explotación pesquera no acogida a las normas, siendo estos los principales problemas que enfrenta el ambiente. Por tanto, la educación ambiental, como proceso educativo para cada individuo, las familias, las comunidades, la sociedad y el Estado debe reorientarse para contribuir al logro del desarrollo sostenible, de esta manera Córdoba (2002):

La educación ambiental es un excelente proceso que, satisfactoriamente enfocado, programado, ejecutado y evaluado en los distintos grupos y sectores de nuestras sociedades, puede renovar y estimular los procesos políticos, económicos, sociales y culturales de nuestros países para contribuir a minimizar la pobreza, el analfabetismo, la falta de educación, carencia de servicios de salud y otros problemas globales contemporáneos, incluyendo los problemas del medio ambiente mundial (pág. 85).

Bajo esta perspectiva, se evidencia que se necesita hoy más que nunca una voluntad e interés de una política que logre priorizar el desarrollo de la Triada: protección del medio ambiente, desarrollo sostenible y educación ambiental como parte de la vida política, económica, social e histórica-cultural de cada nación, para elevar la calidad de vida y el bienestar del desarrollo humano. Uno de los problemas ambientales que ha causado graves deterioros naturales ha sido la deforestación, con mayor impacto en las cabeceras de los ríos, lo que produce sequía en estos, deteriorando cualquier ecosistema que forman parte de las fuentes hídricas.

Por lo tanto, reforestar, se vuelve pues, además de una práctica escolar y educativa, un compromiso moral y social tanto de los estudiantes como de quienes están en contacto directo con el proceso de enseñanza; ya que además de la transmisión de conocimientos, se debe ser lo suficientemente 
capaz de transmitir valores y normas de convivencia tanto con los propios seres humanos como con la naturaleza. Así, según Portillo, (2003), dice:

Reforestar no se limita a la simple plantación masiva y anárquica de árboles, sino que implica una amplia gama de aspectos a considerar, entre los cuales se puede citar de momento, la cultura del cuidado de la vegetación ya existente, el cuidado de los árboles que se han de plantar, reflexionar acerca de la importancia de transmitir un mejor mundo a las próximas generaciones, hacer que las actividades ecológicas se conviertan en situaciones cotidianas del quehacer humano, en fin, hacer de la reforestación un aspecto toral de la educación en particular, y de la vida en general. (pág. 143).

En tal sentido, el ambiente sufre fuertes impactos de las actividades del hombre; donde el $24 \%$ de los sistemas biogeográficos de la Tierra han sido completamente transformados por este y sólo quedan bien conservados el $51,9 \%$ y nivel mundial, más de 20 millones de hectáreas de bosques se pierden anualmente, sin incluir las grandes extensiones que son degradadas por prácticas forestales sin control.

En los actuales momentos, Venezuela se ubica entre los primeros diez países con mayor biodiversidad del planeta y sexto en América. El país también posee unas 15 mil plantas y una enorme variedad de hábitats que incluyen arrecifes coralinos, sabanas, tepuyes, morichales, entre otros.

Además, cuenta con una extensa red de áreas protegidas, representadas fundamentalmente por Parques Nacionales, Monumentos Naturales y Refugios de Fauna Silvestre. Lamentablemente Venezuela, posee una de las tasas de deforestación más altas de América Latina, debido principalmente a la expansión de la frontera agropecuaria y al crecimiento incontrolado de las zonas urbanas. El deterioro del ambiente durante los últimos años se ha visto agravado por las invasiones ilegales de tierra e incluso en áreas protegidas, lo que demuestra que nuestro país está perdiendo su vegetación original a una alta velocidad. 
De manera tal, que conservar el ambiente significa usar de forma racional los recursos naturales, para lograr un desarrollo sostenible que garantice que las generaciones futuras puedan disfrutar de los recursos naturales de la misma manera de quienes habitan en este momento el mundo.

Es por ello que debido a esta situación de desequilibrio ecológico, la reforestación juega un papel elemental en la educación de los jóvenes del país, puesto que esta y sus componentes que contemplan la siembra de árboles para producción o para proteger el medio ambiente tienen impactos ambientales positivos; donde los productos forestales de la reforestación benefician al hombre en tanto que de estos se pueden extraer: madera, pulpa de celulosa, postes, fruta, fibras y combustibles, las arboledas comunitarias y los árboles que siembran agricultores alrededor de sus viviendas o terrenos.

Las plantaciones ofrecen la mejor alternativa a la explotación de los bosques naturales para satisfacer la demanda de madera y otros productos combustibles. Las plantaciones que se realizan para la producción de madera, generalmente emplean las especies de crecimiento más rápido y el acceso y la explotación son más fáciles que en el caso de los bosques naturales pues dan productos más uniformes y comercializables. Asimismo, las plantaciones comunitarias para la producción de leña y forraje, cerca de los poblados, facilitan el acceso de los usuarios a estos bienes y, a la vez, ayudan a aliviar la presión sobre la vegetación local, que puede ser la causa del corte y pastoreo excesivo.

Es importante destacar la importancia de las plantaciones en la recuperación de las áreas verdes, las cuales son importantes para la recreación y el esparcimiento, considerando que ambos satisfacen necesidades humanas básicas de jugar, relacionarse, hacer deporte, disfrutar actividades al aire libre las cuales contribuyen al desarrollo humano integral.

En este sentido Cantor (2008), ha señalado que:

Las áreas de recreación son lugares, que contribuyen a mejorar 
la calidad de vida de los habitantes de determinado lugar, se convierten en enclaves perfectas para poder luchar contra el estrés y la ansiedad, del mismo modo, que fomentan la creatividad de los individuos, de igual manera les permiten interactuar con la naturaleza creando con ello una conciencia, en favor de la conservación del ambiente (pág. 163).

Es por ello que la recuperación de estos espacios de recreación y esparcimiento, conducen a una toma de conciencia real de las condiciones y exigencias que el ambiente requiere para ser adaptado a los procesos humanos sin alterarlo, puesto que la creación Provee un cambio de ritmo de la vida cotidiana. Se refresca el individuo permite mantener un equilibrio de sus dimensiones físicas, mentales, emocionales, sociales y espirituales. Contribuye a una vida satisfaciente, disfrutable y abundante.

Por lo tanto, el estado Barinas no es ajeno, a la problemática que surge como amenaza de manera global, como lo es la deforestación, la cual ha traído como grave efecto, la decadencia de la capa vegetal, evaluándose que este es uno de los estados de nuestro país, con un índice en la obtención de madera, y la tala indiscriminada, el cual ha ocasionado, el debilitamiento de la capa terrestre y por ende la extinción de los bosque, dando con ello origen a la desertificación de las áreas verdes de la región. En este sentido esta realidad es observable en la Comunidad Morrocoy abajo sector I, Parroquia San Silvestre, Municipio Barinas, Estado Barinas, donde se aprecia la desaparición de especies forestales y ornamentales que brinden protección a los espacios de recreación y esparcimiento en las diferentes zonas de la comunidad; en consecuencia esta necesidad, simplifica el accionar orientado la investigación hacia el desarrollo de un programa de reforestación como alternativa ambiental en la recuperación de espacios de recreación y esparcimiento, todo en la búsqueda de fomentar la subsistencia de las especies arbóreas y generar un bienestar social en la comunidad. 


\section{Teoría y Conceptos}

\subsection{Impactos Ambientales de la Reforestación}

Las reforestaciones y sus componentes que contemplan la siembra de árboles para producción o para proteger el medio ambiente tienen impactos ambientales positivos y también negativos. Los productos forestales de la reforestación incluyen: madera, pulpa de celulosa, postes, fruta, fibras y combustibles, las arboledas comunitarias y los árboles que siembran agricultores alrededor de sus viviendas o terrenos. Las actividades orientadas hacia la protección incluyen los árboles sembrados a fin de estabilizar las pendientes y fijar las dunas de arena, las fajas protectoras, los sistemas de agro forestación, las cercas vivas y los árboles de sombra.

En lo que respecta al impacto ambiental de la reforestación puede mencionarse los aspectos positivos y negativos, se tiene que este proceso, en algunos casos produce plantaciones de árboles de crecimiento rápido y ciclo corto los cuales pueden ocasionar el agotamiento de los alimentos del suelo y reducir la fertilidad del espacio donde se encuentran las plantaciones.

\section{2. Áreas Verdes}

Según Nilson (2010), Las áreas verdes han estado adquiriendo una relevancia directamente proporcional al crecimiento de la población en las ciudades. En Venezuela, alrededor del $85 \%$ de la población vive en medios urbanos, lo que significa, un mayor consumo de energía y recursos naturales, lo que trae consigo muchas consecuencias ambientales, sociales y económicas adversas, entre otros problemas que afectan la calidad de vida de una comunidad.

Algunos países, mantienen rezagos importantes en la dotación integral de servicios, lo que incrementa desigualdades y propicia problemas propios de las grandes urbes; por tal razón, en la gran mayoría de los casos, están directamente relacionados a la falta de espacios verdes. Mejorar las 
condiciones de vida en las áreas urbanas, es una tarea prioritaria para diversos sectores tanto gubernamentales como civiles, los cuales reconocen que el manejo sostenible de las áreas verdes urbanas contribuye al bienestar ambiental, social y económico de las sociedades urbanas y debe ser una parte indispensable de cualquier estrategia ambiental del desarrollo sustentable y sostenible de las ciudades.

\subsection{Importancia de las Áreas Verdes para la Biodiversidad y el Ambiente.}

Según Owens (2010), la naturaleza que crea el hombre es considerada con frecuencia inferior a la naturaleza que evoluciona sin intervención humana. Como confirmación de esto se aduce con frecuencia, por ejemplo, que el número de especies suele ser mayor en la naturaleza inalterada. Una investigadora inglesa, que es propietaria de un jardín de unos $700 \mathrm{~m}$ en un suburbio de Leicester, en Midlands, tiene la opinión de que éste no es invariablemente el caso.

Durante 15 años ha recogido y clasificado las especies de todos los insectos que ha encontrado en su jardín. Ha recibido por ejemplo visitas del $34 \%$ de todas las especies indígenas de mariposas, el $30 \%$ de todas las especies indígenas de mariposas nocturnas y el $36 \%$ de todas las especies indígenas de Syrphus. Aunque su jardín es muy corriente tiene unos macizos de flores extraordinariamente tupidos y una buena mezcla de plantas cultivadas y no cultivadas. Sin embargo, muchas áreas verdes urbanas no presentan una biodiversidad particularmente rica. La mayoría de ellas se establecieron con grandes superficies pavimentadas, áreas engravadas, verdes bien segados y árboles individuales aislados.

De acuerdo con Nowak (2011), los árboles interceptan partículas de materia y absorben contaminantes gaseosos como el ozono, el dióxido de azufre y el dióxido de nitrógeno, eliminándolos así de la atmósfera. Los árboles emiten también diversos compuestos orgánicos volátiles como el isopreno y 
los mono terpenos que contribuyen a la formación de ozono en las ciudades. Mediante la transpiración del agua y las superficies sombreadas, los árboles reducen la temperatura del aire. Concluye que debido a que los árboles reducen la temperatura del aire, dan sombra a los edificios en verano y frenan los vientos invernales, pueden contribuir a reducir el consumo de energía en los edificios y en consecuencia a reducir la emisión de contaminantes de las instalaciones generadoras de energía.

Las plantaciones de protección a lo largo de carreteras de tráfico denso y alrededor de las áreas industriales son, por lo tanto, un medio eficaz para reducir la contaminación del aire. Pero esto, indudablemente no puede tomarse como excusa para pasar por alto la lucha contra la contaminación en su origen. Aunque las plantas absorben dióxido de carbono y producen oxígeno, es importante no asignar a las plantas una importancia excesiva para el ambiente urbano.

\subsection{Importancia de las plantas para los seres vivos}

Según Soler (2012), las plantas resultan extremadamente útiles. Por una parte, aportan el oxígeno necesario para poder respirar. Pero además aportan nutrientes para la alimentación. Los vegetales son imprescindibles pues aparte de regenerar el oxígeno que se respira, los seres vivos proporcionan alimentos y materias primas para la industria y muchos otros beneficios, como el de fijar el suelo para que la tierra no se convierta en desierto infértil.

\subsection{Plantas Ornamentales}

Según Fernández (2012), Una planta ornamental es aquella que se cultiva y se comercializa con la finalidad principal de mostrar su belleza, hay numerosas plantas que tienen un doble uso, alimentario y ornamental como el olivo y el naranjo. 
Las plantas ornamentales vivas son aquellas que se venden con o sin maceta pero que están preparadas para ser trasplantadas o simplemente transportadas al lugar de destino. En agricultura las plantas ornamentales normalmente se cultivan al aire libre en viveros o con una protección ligera bajo plásticos o en un invernadero con calefacción o temperatura controlada. La importancia de las plantas ornamentales se ha incrementado con el desarrollo económico de la sociedad y el incremento de las áreas ajardinadas en las ciudades, y con el uso de plantas de exterior e interior por los particulares. Actualmente hay más de 3.000 plantas que se consideran de uso ornamental.

\subsection{La Educación Ambiental}

En lo relativo a la educación ambiental existe amplitud en cuanto a su definición e importancia en la conservación del ambiente, se considera que es nueva forma que facilita la formación de los individuos, donde este es capaz de entender lo complejo que resulta el equilibrio del ambiente, en todas sus facetas, de allí la importancia de transmitir de diversa manera la información, relativa a su funcionamiento de igual manera esta formación va a contribuir, a la formación de un ser más crítico, que le permitirá adoptar nuevas formas de comportamiento con el entorno que le rodea, de igual manera la educación ambiental, permite desarrollar valores, de allí la importancia de forman en las instituciones, tomando en consideración la aplicabilidad de las diversas estrategias, que para ello se requiera.

\section{Metodología}

En el análisis del marco que determina el presente estudio, es pertinente aclarar que según Ruiz (2008), "la naturaleza de una investigación comprende la manera de hacer ciencia, adoptada por la comunidad científica, en un momento histórico determinado" (pág. 9); es decir, supone un conjunto 
de ideas, creencias y estructuras conceptuales que permiten interpretar la realidad; una manera o método particular de indagar dicha realidad y un conjunto de problemas específicos.

De esta manera, La presente investigación asume la metodología cualitativa, propia de las ciencias sociales, la cual en opinión de Taylor y Bogdan (2002), la investigación cualitativa se refiere a "la investigación que produce datos descriptivos: la propias palabras de las personas, habladas 0 escritas y la conducta observable" (pág. 20), por cuanto se examinan de forma analítica la participación en el desarrollo socioeconómico para el prosperidad de la Comunidad Morrocoy Abajo sector I, Parroquia San Silvestre, Municipio Barinas, Estado Barinas.

Desde la perspectiva del paradigma cualitativo, es inaceptable desligar pensamiento y realidad, se tiene la convicción sobre una realidad modelada y construida por los pensamientos, donde se investiga; de acuerdo con Goetz (2000), resalta, "como se forma parte de esa realidad, desde la perspectiva y posibilidad para conocerla, así entre el sujeto de la investigación y el objeto que habla se establece una relación de interdependencia e interacción" (pág. 36). Por tanto, se considera la investigación cualitativa porque su foco de atención coincide en las descripciones detalladas de situaciones, eventos, personas, interacciones y comportamientos que son observables, incorporando la voz de los participantes, sus experiencias, actitudes, creencias, pensamientos y reflexiones tal y como son expresados por ellos mismos.

Bajo este contexto de la investigación cualitativa se define el método de investigación, donde cabe destacar que en el presente estudio empleó el método etnográfico por considerar que es un método de recopilación descriptiva que aborda la realidad de la problemática existente en la investigación planteada, en este sentido Woods (2007), la define como el método de investigación por el que "se aprende el modo de vida de una unidad 
social concreta, pudiendo ser ésta una familia, una clase, un claustro de profesores o una escuela" (pág. 84). Se utiliza para referirse a la descripción del modo de vida de un grupo de individuos. Es quizá el método más conocido y utilizado para analizar la práctica de conductas sociales, describirla desde el punto de vista de las personas que en ella participan y aproximarse a una situación social. Según la complejidad de la unidad, el método etnográfico, establece un continuum entre las macro etnografías, que persiguen la descripción e interpretación de sociedades complejas, hasta la micro etnografía, cuya unidad social viene dada por una situación social concreta.

Por consiguiente, en cuanto a las etapas a cumplirse se atenderá a lo planteado por Martínez (2013), el cual considera:

Etapa Previa: En la misma se seleccionarán los actores sociales, las técnicas de recolección de datos, técnicas de análisis de la información, documentación teórica (referencias y estudios previos).

Etapa descriptiva: constituye en el análisis de las entrevistas y observaciones a fin de destacar los indicadores más importantes que explican el estudio, así como la comparación entre las diversas opiniones, para contrastar la realidad latente.

Etapa Estructural: se enfocará en la interpretación de las categorías, para lo cual se hará uso de la técnica de la reducción, la cual, permitirá seleccionar los aspectos más relevantes de los informantes, estableciendo las semejanzas y diferencias entre los mismos.

Discusión de Resultados: una vez procesada parte de la información, se procederá a la aplicación de triangulaciones, que determinarán las coincidencias de la problemática en estudio, para poder emitir juicios de valor en relación al estudio, estos son, interpretaciones, relaciones, análisis, teorización, entre otros. 


\subsection{Informantes}

Para Taylor (1987), el informante es una persona capaz de aportar información sobre el elemento de estudio, además de ser un informante clave, es una persona que sitúa en el campo y ayuda en el proceso de selección de participantes en el caso de realizar entrevistas o grupos focales. Ambos conceptos también provienen de la etnografía, siendo el portero la persona que facilitaba la entrada y el informante clave la persona que completaba la generación de información de la observación participante mediante entrevistas informales. Por lo tanto, los informantes claves son personas que permiten a los investigadores cualitativos acercarse y comprender en profundidad la realidad que se quiere estudiar en el ámbito social.

De acuerdo ello los informantes o actores sociales que forman parte de la investigación abordada en la comunidad de Morrocoy abajo sector I se estima (03) participantes los cuales, fueron elegidos a criterio de la investigadora, según los actores que se consideran vinculantes: vecinos conocedores de la materia ambiental, con años de estar en contacto con las fuerzas vivas de la comunidad.

\subsection{Técnica e Instrumento de Recolección de la Información}

Hernández, Fernández y Baptista (2010), indica la existencia de diversos tipos de instrumentos de medición, cada una con características diferentes, por tanto, es necesario aclarar que en una investigación hay dos opciones respectos al instrumento de medición. Elegir un instrumento ya desarrollado y disponible, el cual se adapta a los requerimientos del estudio en particular; construir un nuevo instrumento de medición de acuerdo con la técnica apropiada para ello; un proceso investigativo no tiene validez sin la aplicación sistemáticas de técnicas de recolección de datos, ya que ellas conducen a la constatación del problema planteado. Cada tipo de investigación determinara las técnicas específicas a utilizar y cada técnica establece sus 
herramientas, instrumentos o medios que serán empleados.

Cabe señalar que en la investigación cualitativa usa múltiples y útiles técnicas, sin embargo, para el estudio la autora considera la entrevista de mucha utilidad para el objeto de estudio. De esta manera la técnica de la entrevista se aplicó a tres (3) individuos pertenecientes a la comunidad al objeto de estudio

En cuanto a los instrumentos de registros de las entrevistas, se utilizaron grabadora y hojas de recordatorio. Todo ello con la intención de dar fidelidad a las respuestas y opiniones para una mejor utilización de los datos. Por otro parte, se utilizó la revisión documental con la finalidad de extraer del material escrito información relacionada con la reforestación como alternativa ambiental en la recuperación de espacios de recreación y esparcimiento, a fin de triangularla con otras técnicas de recolección de la información.

Se consigue en Sierra (2003), lo siguiente:

Los hombres no sólo tienen sentidos que les permiten observar los fenómenos sociales en el momento mismo en que se producen, sino que con la estructura expresan por medio de signos estas observaciones... Todo este arsenal de escritos, películas, fotografías, reproducciones de sonidos y objetos de toda clase son documentos y constituyen el objeto de la observación documental (pág. 283).

Este autor realza la técnica de la revisión y análisis documental, al afirmar lo siguiente: en cuanto a la investigación actual, es un complemento indispensable a los demás medios de observación de la realidad social. Lo que aportará a la presente investigación una técnica útil para la recolección de la información.

\subsection{Credibilidad de la Información}

La credibilidad y validez de una investigación, de acuerdo con el señalado por Corbetta (2007), tiene un alto nivel de validez si al observar, 
medir o apreciar una realidad se observa, mide o aprecia esa realidad y no otra, lo que conlleva a que la validez se defina como el grado o nivel en que los resultados de la investigación reflejen una imagen clara y representativa de una realidad situacional dada; constituyendo este hecho la validez interna.

A fin de establecer la validez de la información, se utilizó la triangulación de la información de los actores sociales involucrados en la investigación y de las técnicas manejadas. En este orden Martínez (2008), argumenta:

... la credibilidad de la información puede variar mucho: los informantes pueden mentir, omitir datos o tener una visión distorsionada de las cosas. Será necesario contrastarla con lo de los otros o recogerla en momentos diferentes: la triangulación con distintas fuentes de información y con diversos métodos puede resultar muy valiosa (pág. 120).

La credibilidad de la información recabada se llevó a cabo triangulando la misma, contrastando la información de los tres entrevistados, con lo cual se obtuvo una imagen clara y representativa de la situación dada. Igualmente, se trianguló la información obtenida de las dos técnicas a utilizar, la entrevista y el análisis documental.

\section{Conclusiones}

Como conclusiones finales, se puede hacer mención al hecho de que la investigación se encuentra actualmente en proceso de desarrollo, pudiendo establecer que, las comunidades organizadas, manejan estrategias específicas para la integración de estas, donde la orientación oportuna como un proceso social conlleva a la participación activa y efectiva de los involucrados; de allí, este planteamiento, señala que el proceso de participación comunitaria debe ser pertinente a las necesidades del individuosociedad donde se lleve a cabo. 
Entonces, la direccionalidad de la integración entre los integrantes de una comunidad será permanente y deberá reforzarse progresivamente, porque esta debe modelarse con la participación de la comunidad y servir de apoyo a la misma, manteniendo contacto con todos los actores para mejorar sus servicios y serle de utilidad; en la cual cada miembro de la comunidad debe ser un promotor social para estimular la participación y líder para convertir esta en un espacio de vida y salud acorde con las características propias de dicha comunidad.

Cabe resaltar, la reforestación aporta una serie de beneficios y servicios ambientales. Al restablecer o incrementar la cobertura arbórea, se aumenta la fertilidad del suelo y se mejora su retención de humedad, estructura y contenido de nutrientes (reduciendo la lixiviación, proporcionando abono verde y agregando nitrógeno, en el caso de que las especies utilizadas sean de este tipo), de allí la importancia del presente estudio.

Por otra parte, la cobertura arbórea también ayuda a reducir el flujo rápido de las aguas lluvias, regulando, de esta manera, el caudal de los ríos, mejorando la calidad del agua y reduciendo la entrada de sedimento a las aguas superficiales. Debajo de los árboles, las temperaturas más frescas y los ciclos húmedos y secos moderados constituyen un microclima favorable para los microorganismos y la fauna; ayuda a prevenir la laterización del suelo. Las plantaciones tienen un efecto moderador sobre los vientos y ayudan a asentar el polvo y otras partículas del aire.

Al incorporar los árboles pueden mejorarse las condiciones ambientales, gracias a sus efectos positivos para la tierra y el clima. Finalmente, la cobertura vegetal que se establece mediante el desarrollo de las plantaciones en gran escala y la siembra de árboles, constituye un medio para la absorción de carbono, una respuesta a corto plazo al calentamiento mundial causado por la acumulación de dióxido de carbono en la atmósfera. Es por ello, que el estudio adquiere relevancia educativa, debido a que, al 
promover la siembra de árboles, como parte de un programa forestal educativo, permite la sensibilización tanto en la escuela como en la comunidad donde esta se encuentra.

\section{Referencias}

Cantor, M. (2008). Planificación de Sistemas de gestión Ambiental. Maracay, Venezuela: Editorial.

Corbetta, P. (2007). Metodología y Técnicas de Investigación Social. España: McGraw-Hill.

Córdoba, F. (2002). Fundamentos de la Biodiversidad. Caracas, Venezuela: Editorial Fuentes C.A.

Fernández, A. (2012). Trabajo de investigación "Recuperemos el parque para jugar otra vez". Guayaquil, Ecuador.

Goetz, J. (2000). Etnografía y diseño cualitativo en investigación educativa. Madrid: Morata.

Hernández, R., Fernández C. \& Baptista P. (2010). Metodología de la Investigación. 4ta Edición, México: McGraw-Hill. Recuperado de:

https://es.scribd.com/doc/38757804/Metodologia-de-La-InvestigacionHernandez-Fernandez-Batista-4ta-Edicion

Martínez, M. (2013). Nuevos paradigmas en la investigación. Segunda Edición. Caracas, Venezuela: Editorial Alfa.

Martínez, M. (2008). Ciencia y arte en la metodología Cualitativa. México: Trillas.

Nilson, D. (2010). Aspectos tecnológicos del embellecimiento urbano. Bogotá, Colombia: Instituto geográfico Agustín Codazzi.

Nowak, D. (2011). Remoción de la contaminación del aire a través de árboles urbanos. N.Y. USA: Urban Forestry and Urban Greening.

Owens, T. (2010). Términos generales de un Diagnóstico situacional. Colombia: Editorial Universidad Cooperativa de América. 
Portillo, (2003). Sistemas de Gestión Ambiental. Caracas, Venezuela: Editorial UNIDO.

Sierra, B. (2003). Técnicas de Investigación Social, Teorías y ejercicios. España: Thompson.

Soler, M. (2012). Vida y Recursos naturales. Enciclopedia Terranova, Tomo 2do. Editorial Terranova.

Ruiz, A. (2008). Investigación Cualitativa. Retos e Interrogantes. Venezuela: Planeta.

Taylor, S. \& Bogdan, R. (2002). Introducción a los métodos cualitativos de investigación: la búsqueda de los significados. Barcelona, Madrid: Paidós.

Taylor, S. \& Bogdan, R. (1987). Introducción a los métodos cualitativos. Barcelona, Madrid: Paidós.

Woods, P. (2007). La escuela por dentro. La etnografía en la investigación educativa. Barcelona, Madrid: Paidós/MEC. 


\section{Emérita Molina Contreras}

e-mail: emeritacontreras71@gmail.com

Nacida en Camatuche, Estado Barinas, Venezuela. Cursa estudio de maestría en Educación Ambiente y Desarrollo en la Universidad Pedagógica Experimental Libertador, (UPEL) Núcleo Barinas. Licenciada en Educación Mención: Castellano y Literatura Universidad Nacional Experimental de los Llanos Occidentales Ezequiel Zamora (UNELLEZ) Barinas.

Actualmente se desempeña como Docente de Aula de Educación Primaria. Ha participado en cursos de: Crecimiento Personal e Imagen Corporativa, Juegos Dinámicas y Canciones, Valores y Desarrollo Personal, Caligrafía, Ortografía y Redacción, Manualidades, Proyecto Pedagógico de Aula, Currículo Básico Nacional, Operador de Windows XP, Magia del Periódico. También participo en los Talleres de Sensibilización, Oratoria, Trabajo Social Comunitario y Primeros Auxilios Comunitarios.

El contenido de este manuscrito se difunde bajo una Licencia de Creative Commons Reconocimiento- 